\title{
MPC Formulation of GLC
}

\author{
Masoud Soroush and Costas Kravaris \\ Dept. of Chemical Engineering, The University of Michigan, Ann Arbor, MI 48109
}

During the last decade, there has been a growing interest in developing nonlinear model-based control methods. This interest has led to substantial progress mainly within the two frameworks of model predictive control (MPC) and differential geometric control.

MPC is an optimization-based control methodology which explicitly accounts for process constraints and in general leads to a controller without an analytical form (see Muske and Rawlings (1993) for a recent thorough review of MPC). However, differential geometric control is a feedback linearization-based control methodology which leads to a controller with an analytical form, as in globally linearizing control (GLC) (Soroush and Kravaris, 1992).

An objective of this note is to show that not only are these two apparently different control methodologies closely related, but also in some cases they lead to identical controllers. In particular, this note establishes that the input-output linearizing control laws derived in our previous article (Soroush and Kravaris, 1996) are indeed model predictive control laws. The specific objectives of this work are:

- To derive a nonlinear MPC law with the shortest useful prediction horizon for each controlled output.

- To prove that the derived model predictive controller is exactly the reduced-order error-feedback globally linearizing controller derived in (Soroush and Kravaris, 1996).

Following the description of the scope of this work, the shortest-horizon MPC law is derived and shown to be exactly a reduced-order error-feedback globally linearizing controller. The nonlinear MPC law is then applied to unconstrained linear processes, and the resulting linear controller is shown to be exactly a model algorithmic controller (Mehra and Rouhani, 1980) and an internal model controller (Garcia and Morari, 1985).

\section{Scope}

The focus of this study is on the nonlinear, square (equal number of inputs and outputs), multivariable processes described by a mathematical model of the form

$$
\left\{\begin{aligned}
x(k+1) & =\Phi[x(k), u(k)] \\
y(k) & =h[x(k)]
\end{aligned}\right.
$$

Correspondence concerning this article should be addressed to $M$. Soroush at his current address: Dept. of Chemical Engineering, Drexel University, Philadelphia, PA 19104 . where $x=\left[\begin{array}{llll}x_{1} & \ldots & x_{n}\end{array}\right]^{T}, u=\left[\begin{array}{lll}u_{1} & \ldots & u_{m}\end{array}\right]^{T}$, and $y=\left[\begin{array}{lll}y_{1} & \ldots & y_{m}\end{array}\right]^{T}$ denote the vectors of state variables, manipulated inputs, and controlled outputs respectively, all in the form of deviation variables; $\Phi(x, u)$ and $h(x)$ are analytic vector functions.

For a given process, a nonlinear discrete-time model of the form of Eq. 1 can be obtained via approximate time-discretization of a nonlinear continuous-time model, or directly by using an identification method (see e.g., Hernandez and Arkun, 1992). A numerical integration method (e.g., Euler's method, modified Euler's method, or Runge-Kutta-Gill method) can be used to obtain an approximate time-discretization of a continuous-time model. The error resulting from an approximate time-discretization can be viewed as a model error in a nonlinear discrete-time controller synthesis, requiring the resulting controller to be sufficiently robust.

\section{Nonlinear Model Predictive Controller Synthesis}

The derivation of the shortest-horizon MPC law, which is along the lines of that of a linear multivariable model algorithmic controller is described here.

\section{Shortest prediction horizon}

For a controlled output $y_{i}$, the smallest number of sampling periods after which a manipulated input affects the output $y_{i}$, is exactly the relative order of the controlled output $y_{i}$.

Definition 1. For a system of the form of Eq. 1, the relative order of an output $y_{i}$ with respect to the manipulated input vector $u$ is the smallest integer $r_{i}$ for which $y_{i}\left(k+r_{i}\right)$ depends explicitly on the control move $u(k)$ (Nijmeijer and van der Schaft, 1990). If such an integer does not exist, we say that $r_{i}=\infty$. Equivalently, the relative order $r_{i}$ is the smallest integer for which

$$
h_{i} \circ \underbrace{\Phi \bigcirc \ldots \circ \Phi}_{r_{i} \text { times }}
$$

depends explicitly on $u$. Here the notation $O$ represents the usual composition of functions in $x$ : e.g., $\Phi(x, u) \circ \Phi(x, u)=$ $\Phi[\Phi(x, u), u]$. Given a mathematical model of the form of Eq. 1, it is straightforward to calculate every $r_{i}$ by using the formula given in Soroush and Kravaris (1996). 
As a result of the definition of the relative orders $r_{1}, \ldots$, $r_{m}$, we can define the notation

$$
\begin{aligned}
& h_{i}^{0}(x) \stackrel{\Delta}{=} h_{i}(x) \\
& h_{i}^{\mathcal{Q}}(x) \stackrel{\Delta}{=} h_{i}^{\ell-1}[\Phi(x, u)], \quad \ell=1, \ldots, r_{i}-1
\end{aligned}
$$

Using this notation and the definition of the relative order $r_{i}$, we see that there is a manipulated input $u_{j}$ such that

$$
\frac{\partial}{\partial u_{j}} h_{i}^{r_{i}-1}[\Phi(x, u)] \neq 0
$$

and

$$
\begin{aligned}
& y_{i}(k+\ell)=h_{i}^{\ell}(x(k)), \quad \ell=0, \ldots, r_{i}-1 \\
& y_{i}\left(k+r_{i}\right)=h_{j}^{r_{i}-1}\{\Phi[x(k), u(k)]\}
\end{aligned}
$$

Thus, the relative order $r_{i}$ is the smallest number of sampling periods after which a manipulated input $u_{j}$ affects the output $y_{i}$; it is the shortest useful prediction horizon for the output $y_{i}$.

If a system output $y_{i}$ does not have a finite relative order ( $r_{i}$ is not finite), this means that none of the manipulated inputs $u_{1}, \ldots, u_{m}$ affect the output $y_{i}$ ( $y_{i}$ is not controllable). Throughout this note, it is assumed that all the relative orders are finite, and locally there is no singular point at which $\partial\left\{h_{i}^{r_{i}-1}[\Phi(x, u)]\right\} / \partial u=[0 \ldots 0], i=1, \ldots, m$.

\section{Output prediction}

For a process with a mathematical model of the form of Eq. 1, the future values of each controlled output $y_{i}$ can be calculated by using the model. The predicted values of an output $y_{i}$ up to $r_{i}$ sampling periods ahead are obtained by adding the measured output signal $\bar{y}_{i}(k)$ to the future changes in the output $y_{i}$, predicted by the model (i.e., Eq. 2)

$$
\begin{gathered}
\hat{y}_{i}(k+\ell) \stackrel{\Delta}{=} \bar{y}_{i}(k)+\underbrace{h_{i}^{\ell}(x(k))-h_{i}(x(k))}_{\left\{\begin{array}{l}
\text { future change in } y_{i} \\
\text { predicted by model }\}
\end{array}\right.}, \quad \ell=1, \ldots, r_{i}-1 \\
\hat{y}_{i}\left(k+r_{i}\right) \stackrel{\Delta}{=} \bar{y}_{i}(k)+\underbrace{h_{i}^{r_{i}-1}(\Phi(x(k), u(k)))-h_{i}(x(k))}_{\left\{\begin{array}{l}
\text { future change in } y_{i} \\
\text { predicted by model }
\end{array}\right\}}
\end{gathered}
$$

where $\hat{y}_{i}$ denotes the predicted output. The vector of state variables $x$ will be calculated by using a full-order open-loop state-observer (by on-line simulation of the process model). Because a full-order open-loop state-observer can only be used for open-loop stable processes, the use of an observer of this type limits the class of processes to which the derived controller will be applicable.

\section{Reference trajectories: setpoint filters}

Reference trajectory of an output $y_{i}$, denoted by $y_{d_{i}}$, is the trajectory which the model predictive controller will try to force the output $y_{i}$ to follow, in the absence of constraints and penalties on the controller action. Because we intend to derive a shortest prediction-horizon MPC law, we have to use reference trajectories so that the resulting model predictive controller is robust. Otherwise, the controller will be exactly an output deadbeat controller (Glad, 1987), which is known for its poor robustness.

As in linear model algorithmic control (Mehra and Rouhani, 1980), for each controlled output $y_{i}$, a linear reference trajectory $y_{d_{i}}$ is defined

$$
y_{d_{i}}\left(k+r_{i}\right)=y_{s p_{i}}(k)+\sum_{j=1}^{m} \alpha_{i j}\left[\hat{y}_{j}\left(k+r_{j}-1\right)-y_{s p_{j}}(k)\right]
$$

where $\alpha_{i j}, i, j=1, \ldots, m$, are tunable scalar parameters. Thus, the reference trajectories are governed by

$$
\left[\begin{array}{c}
y_{d_{1}}\left(k+r_{1}\right) \\
\vdots \\
y_{d_{m}}\left(k+r_{m}\right)
\end{array}\right]=\left\{I_{m}-\alpha\right\}_{s p}(k)+\alpha\left[\begin{array}{c}
\hat{y}_{1}\left(k+r_{1}-1\right) \\
\vdots \\
\hat{y}_{m}\left(k+r_{m}-1\right)
\end{array}\right]
$$

where $\alpha=\left[\alpha_{i j}\right]$ is an $m \times m$ matrix. In the case that the matrix $\alpha=0$ and the controller action is neither penalized nor bounded, resulting model predictive controller will be an output deadbeat controller.

\section{Optimization problem - derivation of the control law}

We consider a minimization problem of the form

$$
\min _{u(k)}\left\{\sum_{j=1}^{m} \theta_{j}\left[y_{d_{j}}\left(k+r_{j}\right)-\hat{y}_{j}\left(k+r_{j}\right)\right]^{2}+\sum_{j=1}^{m} \rho_{j} u_{j}^{2}(k)\right\}
$$

subject to the input constraints

$$
u_{l_{\ell}} \leq u_{\ell}(k) \leq u_{h_{\ell}}, \quad \ell=1, \ldots, m
$$

where $\theta_{1}, \ldots, \theta_{m}, \rho_{1}, \ldots, \rho_{m}$ are positive tunable parameters, $\hat{y}_{\ell}\left(k+r_{\ell}\right)$ and $y_{d_{\ell}}\left(k+r_{\ell}\right)$ are given by Eqs. 3 and 5 , and $x$ is obtained by simulating the process model $x(k+1)=$ $\Phi[x(k), u(k)]$.

\section{Unconstrained processes}

When there is no input constraint and the controller action is not penalized, the minimization problem of Eq. 6 takes the form

$$
\min _{u(k)}\left\{\sum_{j=1}^{m} \theta_{j}\left[y_{d_{j}}\left(k+r_{j}\right)-\hat{y}_{j}\left(k+r_{j}\right)\right]^{2}\right\}
$$

In this case, the resulting controller tries to minimize a weighted sum of the squared, predicted mismatches between the controlled outputs and the reference trajectories. As a special case, we seek a controller which can force every controlled output $y_{i}$ to follow the corresponding reference trajectory $y_{d_{i}}$ exactly (which makes the performance index of Eq. 7 zero) 


$$
\left[\begin{array}{c}
y_{d_{1}}\left(k+r_{1}\right)-\hat{y}_{1}\left(k+r_{1}\right) \\
\vdots \\
y_{d_{m}}\left(k+r_{m}\right)-\hat{y}_{m}\left(k+r_{m}\right)
\end{array}\right]=0
$$

As we will see, such a controller exists only for a certain class of processes. The required control move $u(k)$, which makes the performance index in Eq. 7 zero, is the solution of the following system of nonlinear algebraic equations (using Eqs. 3 and 5)

$$
\begin{aligned}
& {\left[\begin{array}{c}
h_{1}^{r_{1}-1}\{\Phi[x(k), u(k)]\} \\
\vdots \\
h_{m}^{r_{m}-1}\{\Phi[x(k), u(k)]\}
\end{array}\right]} \\
& \quad=\left(I_{m}-\alpha\right)\{e(k)+h[x(k)]\}+\alpha\left[\begin{array}{c}
h_{1}^{r_{1}-1}[x(k)] \\
\vdots \\
h_{m}^{r_{m}-1}[x(k)]
\end{array}\right]
\end{aligned}
$$

where the error $e=y_{s p}-\bar{y}$. The preceding algebraic equations are, in general, nonlinear in $u$ and may not have a solution for $u(k)$. To ensure the existence of a solution to these algebraic equations, we have to identify the class of processes for which such a controller exists.

Definition 2. Consider a discrete-time system of the form of Eq. 1, and assume that each output $y_{i}$ possesses a finite relative order $r_{i}$. The $m \times m$ matrix

$$
\mathcal{C}(x, u) \stackrel{\Delta}{=}\left[\begin{array}{c}
\frac{\partial}{\partial u} h_{1}^{r_{1}-1}[\Phi(x, u)] \\
\vdots \\
\frac{\partial}{\partial u} h_{m}^{r_{m}-1}[\Phi(x, u)]
\end{array}\right]
$$

is called the characteristic matrix of the system (Nijmeijer and van der Schaft, 1990). sary and sufficient condition for the existence of a solution for $u$. Throughout this article, it will be assumed that $\mathfrak{C}(x, u)$ is locally nonsingular, and Eq. 8 has a real solution for $u$ at each time instant.

For a process model of the form of Eq. 1 with a nonsingular characteristic matrix, the implicit function theorem ensures the local existence of a function

$$
u=\Psi_{o}(x, v),
$$

which satisfies the set of algebraic equations

$$
\left[\begin{array}{c}
h_{1}^{r_{1}-1}[\Phi(x, u)] \\
\vdots \\
h_{m}^{r_{m}-1}[\Phi(x, u)]
\end{array}\right]=v .
$$

Using the definition of $\Psi_{o}$ (Eqs. 9 and 10), the solution to the system of algebraic equations of Eq. 8 takes the form

$$
\begin{aligned}
u(k)=\Psi_{o}\left(x(k),\left(I_{m}-\alpha\right)\{e(k)+h[x(k)]\}\right. \\
\left.+\alpha\left[\begin{array}{c}
h_{1}^{r_{1}-1}[x(k)] \\
\vdots \\
h_{m}^{r_{m}-1}[x(k)]
\end{array}\right]\right)
\end{aligned}
$$

which is also the solution to the minimization problem of Eq. 7. Thus, the nonlinear MPC law is given by Eq. 11, where $x(k)$ is obtained by simulating the process model, $x(k+1)=$ $\Phi[x(k), u(k)]$. Equivalently, it can be represented by the following dynamic nonlinear error-feedback control law:

$$
\left\{\begin{array}{c}
x(k+1)=\Phi\left\{x(k), \Psi_{o}\left(x(k),\left(I_{m}-\alpha\right)\{e(k)+h[x(k)]\}+\alpha\left[\begin{array}{c}
h_{1}^{r_{1}-1}[x(k)] \\
\vdots \\
h_{m}^{r_{m}-1}[x(k)]
\end{array}\right]\right)\right\} \\
u(k)=\Psi_{o}\left(x(k),\left(I_{m}-\alpha\right)\{e(k)+h[x(k)]\}+\alpha\left[\begin{array}{c}
h_{1}^{r_{1}-1}[x(k)] \\
\vdots \\
h_{m}^{r_{m}-1}[x(k)]
\end{array}\right]\right)
\end{array}\right.
$$

A necessary condition for existence of a solution to Eq. 8 is that the process deadtimes should be balanced (Soroush, 1996). This condition can be expressed in terms of the differential geometric notion of characteristic matrix; a necessary condition for the existence of a solution to Eq. 8 is that the characteristic matrix of the process should be generically nonsingular (Soroush, 1996). Note that in the case of linear systems, nonsingularity of the characteristic matrix is a neces-

\section{Constrained processes}

In the case of a process with active input constraints, in contrast to the single-input single-output case (Soroush and Kravaris, 1992), the constrained minimization problem of Eq. 6 , in general, does not have an analytical solution; the minimization problem should be solved numerically on-line to calculate the exact optimal controller action. An approximate 
analytical solution to the constrained minimization problem, however, can be obtained by calculating the unconstrained controller action (i.e., solution to the unconstrained minimization problem) and then (a) "clipping" the calculated unconstrained controller action or (b) scaling down the unconstrained controller action while maintaining its direction (Campo and Morari, 1990).

In the case that (i) a rearrangement of manipulated inputs or controlled outputs makes the characteristic matrix of the process model diagonal (process is completely or strongly input-output decoupled), and (ii) a diagonal $\alpha$ is chosen, the exact solution to the constrained minimization problem may be obtained simply by clipping the unconstrained controller action.

In the absence of the input constraints, the minimization problem of Eq. 6 has an analytical solution: the minimizing $u(k)$ is the solution to the system of the nonlinear algebraic equations

$$
\begin{aligned}
\left.\operatorname{ddiag}\left\{\rho_{i}\right\}\right] u(k)+\{\mathfrak{e}[x(k), u(k)]\}^{T}\left[\operatorname{diag}\left\{\theta_{i}\right\}\right] \\
\cdot\left\{\left[\begin{array}{c}
h_{1}^{r_{1}-1}\{\Phi[x(k), u(k)]\} \\
\vdots \\
h_{m}^{r_{m}-1}\{\Phi[x(k), u(k)]\}
\end{array}\right]-\left(I_{m}-\alpha\right)\{e(k)+h[x(k)]\}\right. \\
\left.-\alpha\left[\begin{array}{c}
h_{1}^{r_{1}-1}[x(k)] \\
\vdots \\
h_{m}^{r_{m}-1}[x(k)]
\end{array}\right]\right\}=0
\end{aligned}
$$

where $x(k)$ is obtained by simulating the process model, $x(k$ $+1)=\Phi[x(k), u(k)]$. In this case, because the controller action is penalized, the controller cannot force the controlled outputs to follow the linear reference trajectories. Thus, this controller is not input-output linearizing.

\section{Input-output linearizing MPC law}

In the case of a process with a perfect model and a nonsingular characteristic matrix, under a proper initialization of process model [i.e., $x(0)=\bar{x}(0)$ ], the model predictive controller of Eq. 12 forces every controlled output to follow the corresponding reference trajectory exactly; that is, the outputs will evolve according to the same linear dynamics which govern the reference trajectories

$$
\left[\begin{array}{c}
y_{1}\left(k+r_{1}\right) \\
\vdots \\
y_{m}\left(k+r_{m}\right)
\end{array}\right]-\alpha\left[\begin{array}{c}
y_{1}\left(k+r_{1}-1\right) \\
\vdots \\
y_{m}\left(k+r_{m}-1\right)
\end{array}\right]=\left\{I_{m}-\alpha\right\} y_{s p}(k)
$$

This implies that the model predictive controller induces a linear input-output behavior to the closed-loop system; the model predictive controller is an input-output linearizing controller. Indeed, it is exactly the reduced-order errorfeedback globally linearizing controller of Eq. 37 in Soroush and Kravaris (1996). The error-feedback GLC structure is depicted in Figure 1.

This equivalence implies that an unconstrained MPC law with the shortest useful prediction horizon for each output is

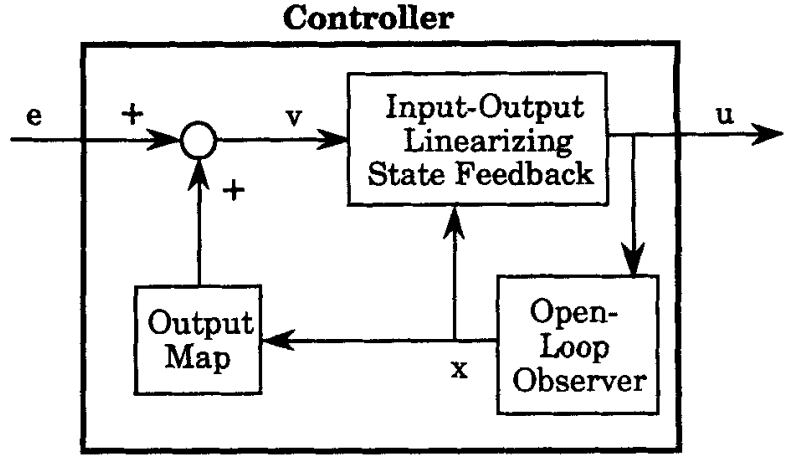

Figure 1. Reduced-order error-feedback GLC structure.

exactly a differential geometric controller. In other words, the reduced-order error-feedback globally linearizing controller is an unconstrained, nonlinear model algorithmic controller with (a) prediction horizons of one sampling period beyond the minimum deadtimes, (b) control horizons of one, (c) linear reference trajectories, and (d) a quadratic performance index with no penalties on controller action.

Remark 1. The dynamic, input-output linearizing errorfeedback controller of Eq. 12 implicitly possesses integral action. Thus, in the presence of an active input constraint, the controller will exhibit windup. This windup can be prevented by updating the state variables of the model (controller) according to

$$
x(k+1)=\Phi\{x(k), \tilde{u}(k)\}
$$

where $\tilde{u}(k)$ is the feasible control action which is actually implemented. The feasible control action $\tilde{u}$ is obtained by clipping or scaling-down the unconstrained controller action calculated from Eq. 11 (see $i$ and ii in the preceding subsubsection). The model predictive formulation of input-output linearizing control led to this solution to the problem of windup in input-output linearizing control. This approach prevents windup by ensuring that at each time instant, the state variables of the controller are driven by the same feasible control action which is actually implemented.

Remark 2. In the case of a constrained process whose characteristic matrix is diagonal (process is completely or strongly input-output decoupled), if a shortest horizon nonlinear MPC with a diagonal $\alpha$ is used, then the exact controller action may be obtained simply by applying the saturation function to each unconstrained manipulated input calculated by the reduced-order error-feedback globally linearizing controller of Eq. 12.

Remark 3. Because the derived unconstrained MPC law of Eq. 12 and the reduced-order error-feedback GLC are identical, the conditions for closed-loop stability under the two control laws are the same. In the absence of constraints and penalties on the controller action, the closed-loop system under the model predictive controller will be input-output stable, if the matrix $\alpha$ is chosen such that all of the eigenvalues of $\alpha$ lie inside the unit circle. For an input-output stable closed-loop system, the local internal closed-loop stability will be ensured, if the process is locally hyperbolically minimumphase and locally asymptotically open-loop stable.

Remark 4. For an unconstrained process with a perfect model, if the matrix $\alpha$ is chosen to be diagonal $\left(\alpha=\operatorname{diag}\left\{\alpha_{i}\right\}\right)$ 
and the controller is initialized appropriately (i.e., $x(0)=$ $\bar{x}(0)$ ), the error-feedback controller of Eq. 12 will induce the completely input-output decoupled, first-order-plus-deadtime response

$$
y_{i}\left(k+r_{i}\right)-\alpha_{i} y_{i}\left(k+r_{i}-1\right)=\left(1-\alpha_{i}\right) y_{s p_{i}}(k), \quad i=1, \ldots, m
$$

to the closed-loop system.

\section{Application to Unconstrained Linear Processes}

Consider a linear, multivariable process described by a discrete-time state-space model of the form

$$
\left\{\begin{aligned}
x(k+1) & =A x(k)+B u(k), \quad x(0)=0, \quad u(0)=0 \\
y(k) & =C x(k)
\end{aligned}\right.
$$

where $A, B$, and $C$ are matrices of dimensions $n \times n, n \times m$, and $m \times n$, respectively. This is a special case of the system of Eq. 1: $\Phi(x(k), u(k))=A x(k)+B u(k) ; h(x(k))=C x(k)$. The input-output behavior of the system of Eq. 15 is described by the $z$-domain matrix-transfer function: $G(z)=$ $C\left(z I_{n}-A\right)^{-1} B$.

Applying Definitions 1 and 2 to the system of Eq. 15, we see that: (a) the relative order $r_{i}$ is the smallest integer for which $c_{i} A^{r_{i}-1} B \neq[0 \ldots 0]$, where $c_{i}$ is the $i$ th row of the matrix $C$; (b) the characteristic matrix (Chen, 1984)

$$
\mathfrak{C}=\left[\begin{array}{c}
c_{1} A^{r_{1}-1} B \\
\vdots \\
c_{m} A^{r_{m}-1} B
\end{array}\right]=\lim _{z \rightarrow \infty}\left[\operatorname{diag}\left\{z^{r_{i}}\right\} G(z)\right],
$$

which is assumed to be nonsingular.

For this lineat case, the function $\Psi_{o}$ has the simple explicit form: $\Psi_{0}(x, v)=\mathfrak{C}^{-1}(v-\mathcal{B} x)$, where $\mathbb{B}=\left[c_{1} A^{r_{1}} \ldots\right.$ $\left.c_{m} A^{r_{m}}\right]^{T}$. Using the algebraic identity

$$
\operatorname{diag}\left\{z^{r_{i}}\right\} C\left(z I_{n}-A\right)^{-1} B=Q B\left(z I_{n}-A\right)^{-1} B+\mathfrak{e}
$$

the matrix-transfer function $G(z)$ can be factored into two parts: (i) a pure delay part $\operatorname{diag}\left\{z^{-r_{i}}\right\}$; and (ii) a "delay-free" part [if $\mathfrak{e}$ is nonsingular],

$$
H(z)=\mathscr{B}\left(z I_{n}-A\right)^{-1} B+\mathcal{C} .
$$

For this case, the model predictive controller of Eq. 12 takes the form

$$
\left\{\begin{aligned}
x(k+1) & =\left\{A-B \mathfrak{C}^{-1}\left[\mathbb{B}-\alpha \mathfrak{D}-\left(I_{m}-\alpha\right) C\right]\right\} x(k) \\
& +B \mathcal{C}^{-1}\left(I_{m}-\alpha\right) e(k) \\
u(k) & =-\mathfrak{C}^{-1}\left[\mathbb{B}-\alpha \mathfrak{D}-\left(I_{m}-\alpha\right) C\right] x(k) \\
& +\mathcal{C}^{-1}\left(I_{m}-\alpha\right) e(k)
\end{aligned}\right.
$$

where $\mathfrak{D}=\left[c_{1} A^{r_{1}-1} \ldots c_{m} A^{r_{m}-1}\right]^{T}$. The system of Eq. 17 is a minimal-order state-space realization of the $z$-domain trans- fer function error-feedback controller (internal model controller)

$$
\begin{aligned}
u(z)=[H(z)]^{-1}\left[I_{m}-\alpha z^{-1}-\left(I_{m}-\alpha\right)\right. & \left.\operatorname{diag}\left\{z^{-r_{i}}\right\}\right]^{-1} \\
& \times\left(I_{m}-\alpha\right) e(z)
\end{aligned}
$$

where $H(z)$, given by Eq. 16 , is the delay-free part of the process model. This error-feedback controller is exactly a multivariable linear internal model controller and a model algorithmic controller, which induce a closed-loop response of the form

$$
y(z)=\operatorname{diag}\left\{z^{-r_{i}}\right\}\left(I_{m}-\alpha z^{-1}\right)^{-1}\left(I_{m}-\alpha\right) y_{s p}(z)
$$

If $\alpha$ is chosen to be a diagonal matrix ( $\alpha=\operatorname{diag}\left\{\alpha_{i}\right\}$ ), the controller of Eq. 17 will be a minimal-order state-space realization of the following internal model controller with a diagonal filter (Garcia and Morari, 1985)

$$
u(z)=[H(z)]^{-1} \operatorname{diag}\left\{\frac{1-\alpha_{i}}{1-\alpha_{i} z^{-1}-\left(1-\alpha_{i}\right) z^{-r_{i}}}\right\} e(z)
$$

which induces the completely input-output decoupled, closed-loop response of Eq. 14.

\section{Acknowledgment}

Financial support from the National Science Foundation through the grant CTS-8912836 is gratefully acknowledged.

\section{Literature Cited}

Campo, P. J., and M. Morari, "Robust Control of Processes Subject to Saturation Nonlinearities," Comput. Chem. Eng., 14, 343 (1990).

Chen, C. T., Linear System Theory and Design, Holt, Rinehart and Winston, New York (1984).

Garcia, E. C., and M. Morari, "Internal Model Control. 2. Design Procedure for Multivariable Systems," Ind. Eng. Chem., 24, 472 (1985).

Glad, S. T., "Output Dead-Beat Control for Nonlinear Systems with One Zero at Infinity," Sys. Cont. Lett., 9, 249 (1987).

Hernandez, E., and Y. Arkum, "Study of the Control-Relevant Properties of Backpropagation Neural Network Models of Nonlinear Dynamical Systems," Comput. Chem. Eng., 16, 227 (1992).

Mehra, R. K., and R. Rouhani, "Theoretical Considerations on Model Algorithmic Control for Nonminimum Phase Systems," Proc. ACC, TA8-B (1980).

Muske, K. R., and J. B. Rawlings, "Model Predictive Control with Linear Models," AIChE J., 39(2), 262 (1993).

Nijmeijer, H., and A. J. van der Schaft, Nonlinear Dynamical Control Systems, Springer-Verlag, New York (1990).

Soroush, M., "Evaluation of Achievable Control Quality in Nonlinear Processes," Comput. Chem. Eng., 20(4), 35 (1996).

Soroush, M., and C. Kravaris, "Discrete-Time Nonlinear Feedback Control of Multivariable Processes," AIChE J., 42(1), 187 (1996).

Soroush, M., and C. Kravaris, "Discrete-Time Nonlinear Controller Synthesis by Input/Output Linearization," AIChE J., 38, 1923 (1992).

Manuscript received July 27, 1995, and revision received Dec. 27, 1995 . 